\title{
Impact Induced Desorption of Large Molecular Structures from Graphitic Substrates
}

\author{
R.P.Webb and K.J.Kirkby \\ Advanced Technology Institute, University of Surrey, Guildford, GU2 7XH, UK, \\ R.Webb@eim.surrey.ac.uk
}

\begin{abstract}
We use molecular dynamics computer simulation to show how the impact of a C60 molecule on a graphite surface can cause an acoustic wave across the surface of the substrate that is strong enough to desorbed a neighboring $\mathrm{C}_{60}$ molecule that has already been adsorbed. This could have potential implications for experimentalists attempting to grow fullerene based structures on graphite like materials using an energetic deposition technique. It suggests that unlike normal growth conditions were sticking probabilities are the principle concern an extra problem of deposition induced desorption may further slow a growth process.
\end{abstract}

Keywords: Computer Simulation, Fullerene Deposition, Graphite

\section{INTRODUCTION}

The phenomenon of sputtering has been well known and understood for over a century now and has been put to great uses in areas such as Secondary Ion Mass Spectrometry (SIMS) and Focused Ion Beam (FIB) lithography techniques, and been the cause of some minor technological problems employing ion beams by causing premature saturation effects in implantation and undesired surface morphology changes in many other applications. In the majority of cases these problems have been overcome relatively simply.

Currently many new applications are being considered which involve the positioning of large fullerene based objects on surfaces to create both active and passive devices. The investigation presented here uses Molecular Dynamics computer simulation [1] to highlight a potential pit-fall that might cause some initial problems when attempting to build these systems using an energetic deposition mechanism particularly if the substrate material is a layered material such as graphite.

We have employed full many body potentials with an additional long range component to model the Van de Waals forces [2] to demonstrate the behaviour of the surrounding surface after a relatively gentle impact of fullerenes (single cage fullerenes $-\mathrm{C}_{60}$ - have been used as well as multiple cage - $\mathrm{C}_{300}$ and $\mathrm{C}_{840}$ ) onto a graphite (hopg) surface. It is seen that the impact, whilst leaving the molecule intact can cause an acoustic wave on the surface of the graphite. Previous work [3] has shown that this acoustic wave can cause substantial ejection of benzene molecules adsorbed onto the surface of the substrate. Here we use the same technique and show that the same acoustic wave can cause desorption of a large molecules as well.

\section{SIMULATION METHOD}

The precise details of the computer simulation technique used in this investigation have been given elsewhere $[1,4]$ hence only a brief summary outlining the important components will be given here. The classical molecular dynamics simulation technique is employed solving Newton's Laws of Motion for many thousands of atoms obeying a realistic many body interaction potentials. The interaction potential used is the Brenner hydrocarbon potential $[5,6]$ with an additional long range term as described by Beardmore and Smith [7]. The elastic properties of this potential have been investigated more fully by Smith and Christopher [8] in a simulation of nanoindentation for diamond, graphite and fullerite films. In their simulations they find good agreement with experiment. In the results presented here the elastic behavior of the graphite surface is quite key in determining the exact results and so it is important that this behavior is modeled well. However it can be expected that the phenomenon discussed here is likely to occur in any layered material, like graphite, to a lesser or greater extent.

The long-range term provides the interlayer bonding term for graphite and the Van der Waals attraction of molecules to the graphite surface. The binding energy of clusters to the graphite surface is calculated by moving the particle close to the surface and away to beyond the cut-off of the long range potential. The potential energy of a fullerene $\mathrm{C}_{60}$ molecule is plotted as a function of distance from the surface and shown in figure 1 . The binding energy is found to be $0.47 \mathrm{eV}$ to the graphite surface at an equilibrium distance of $6.3 \AA$ above the surface, the distance being measure from the center of the cage structure. Previous simulations [9] of binding and equilibrium distances of benzene molecules in this way have found good agreement with experimental values. Similar results are found for $\mathrm{C}_{300}$ structures with a binding energy of $0.94 \mathrm{eV}$ at $10 \AA$ above the surface. Cell sizes contain about 100,000 atoms depending upon the over-layer and projectile simulated. The lateral edges of the simulation cell are treated as periodic but the surface and bottom are treated as free boundaries. The simulations are run for 
typically $4 \mathrm{ps}$, if they are run for much longer then reflections from the periodic boundaries can cause noticeable effects to the results.

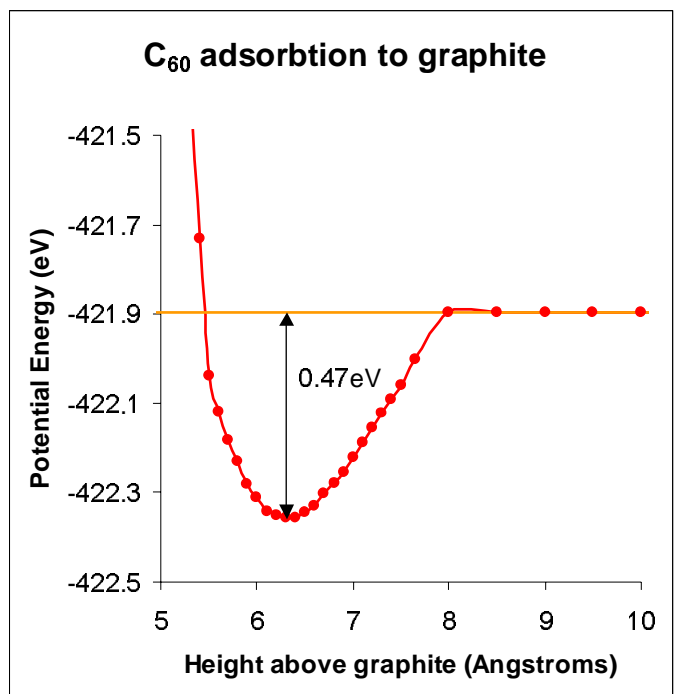

Figure 1: Potential Energy of C60 as a function of height above graphite surface.

\section{RESULTS}

The simulations have been run to observe the effects of the impact from a fullerene molecule on a graphite surface on which a fullerene molecule has been adsorbed. As described above the impact at energies of $200 \mathrm{eV}$ (that is an equivalent energy of $3.33 \mathrm{eV}$ per atom) can cause the propagation of acoustic waves across the surface which have sufficient energy to cause the ejection of small benzene molecules that have previously been adsorbed to the surface. Can the same mechanism cause the ejection of larger adsorbed molecules?

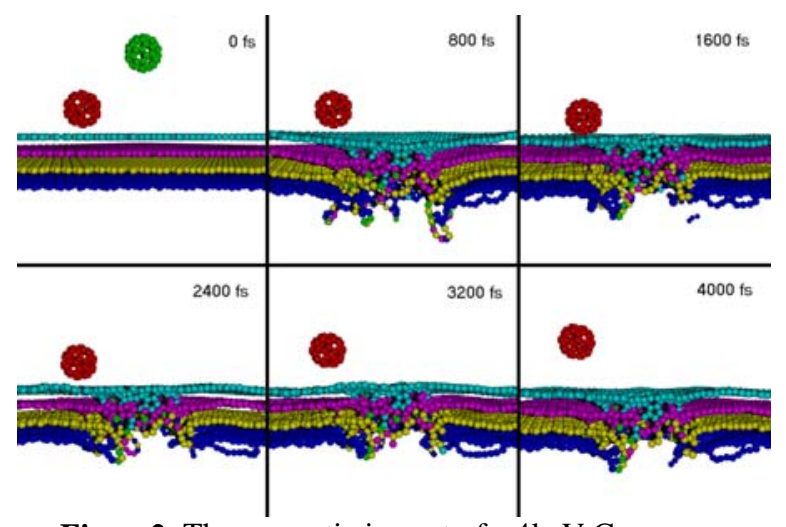

Figure2: The energetic impact of a $4 \mathrm{keV} \mathrm{C}_{60}$ on a graphite surface - see text for discussion.
The figure above shows that an energetic impact of a $\mathrm{C}_{60}$ molecule can cause the desorption of a neighboring $\mathrm{C}_{60}$ molecule. The figure shows the ejection of a nearby adsorbed $\mathrm{C}_{60}$ molecule (drawn in red) caused by the impact of a $4 \mathrm{keV} \mathrm{C}_{60}{ }^{+}$molecule (drawn in green). In the initial frame the "red" $\mathrm{C}_{60}$ is bound to the surface and the impacting "green" $\mathrm{C}_{60}$ is about to make impact with the surface. In this figure the atoms between the "viewing point" and the impact point have been removed from the drawing, but are present in the simulation, to allow the observer a clearer view as to what happens at the impact site and close to the desorbed molecule. In each frame the time after the start of the simulation in fs is shown. It can be seen that the energetic $\mathrm{C}_{60}$ impacts the surface and breaks through the surface of the graphite and destroys itself and causes an acoustic wave to propagate across the surface from the impact site. This wave then interacts with the adsorbed $\mathrm{C}_{60}$ giving it enough energy (at about $2400 \mathrm{fs}$ ) to overcome the binding between it and the surface.

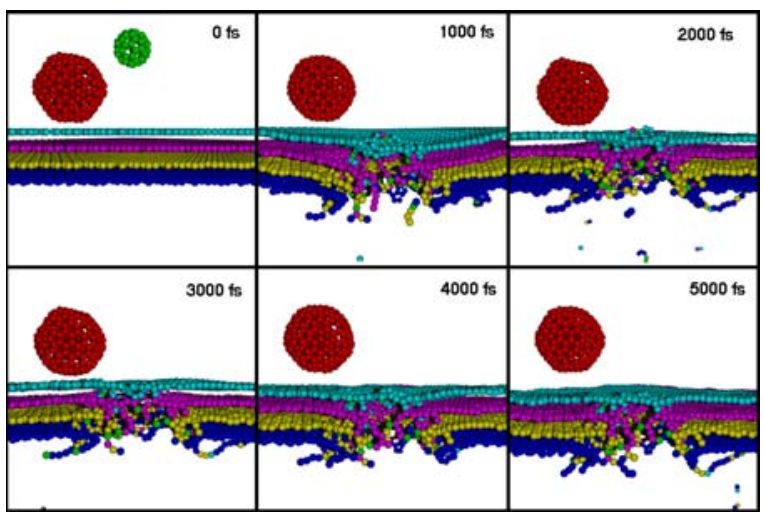

Figure 3: The energetic impact of a $4 \mathrm{keV} \mathrm{C}_{60}$ near a $\mathrm{C}_{300}$ adsorbed molecule

In figure 3 we show a similar set of results for a similar energy impact, but this time near a $\mathrm{C}_{300}$ adsorbed cluster. The effect is very similar despite the increased mass of the cluster and the higher binding. The impacting $\mathrm{C}_{60}$ creates an acoustic wave in the surface of the graphite with enough strength to cause the $\mathrm{C}_{300}$ cluster to be desorbed from the surface. The mechanism is very similar, the initial impact causes the surface to move downwards - away from the adsorbed molecule - pulling it with it. The surface then recovers and springs back, hitting the adsorbed molecule and giving it enough energy to escape the surface binding potential.

These examples have been modeled at a higher energy ( $4 \mathrm{keV}$ for the $\mathrm{C}_{60}$ or $66.67 \mathrm{eV}$ per atom) than would be used in a deposition system it does demonstrate a potential problem of such a technique. Also, whilst the overall energy is relatively high the energy per incoming atom is lower than is often used in a deposition system It should also be noted that fullerene impacts of $250 \mathrm{eV}$ which are enough to "soft-land" a fullerene on the surface also create 
acoustic waves [10]. We have investigated a number of impacts at $250 \mathrm{eV}$ at a range of distances away from the adsorbed fullerene molecule and the general results can be compared in figure 4 below.

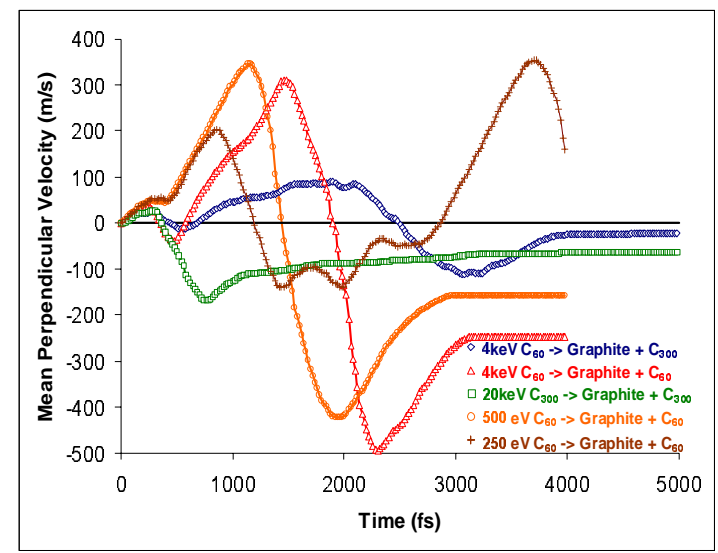

Figure 4: Perpendicular Velocities of "adsorbed" molecule for various fullerene impacts

Figure 4 shows the mean perpendicular velocity of all the atoms in the adsorbed molecule (either a $\mathrm{C}_{300}$ cluster of $\mathrm{a}_{60}$ molecule) as a function of time during the simulation. The same effect can be seen here as were described above from the atom positions. Looking at the red curve on the figure (the $4 \mathrm{keV} \mathrm{C}_{60}$ impact near an adsorbed $\mathrm{C}_{60}$ ). The perpendicular velocity of the adsorbed molecule show that the molecule is first pulled towards the surface (+ve direction) and then pushed away from the surface (-ve direction), this is repeated with more energy and at about $2400 \mathrm{fs}$ the molecule is give enough energy to to escape the surface, which it eventually does at about 3 ps. A very similar effect is seen (orange curve) when the energy of the impacting molecule is dropped to $500 \mathrm{eV}(8.3 \mathrm{eV}$ per atom). When the adsorbed molecule is changed for a $\mathrm{C}_{300}$ cluster (blue curve) then the story is very similar but the velocities are lower. If we change the impacting particle to a $\mathrm{C}_{300}$ cluster as well then the adsorbed cluster is desorbed from the surface with the first vibration of the surface. However in the case of the impact by a $250 \mathrm{eV} \mathrm{C}_{60}$ (brown curve), an energy at which soft-landing occurs, it can be seen that the adsorbed molecule undergoes the same behavior except that when the molecule is pushed away from the surface it does not acquire enough velocity to escape the surface and remains bound. However, the velocity on the second bounce is substantially higher as it comes closer to the surface and at $4 \mathrm{ps}$ it does appear to be about to start moving away from the surface and the curve gives a hint that in the following time-steps the molecule might well shake itself free of the surface. Unfortunately the simulation time beyond $4 \mathrm{ps}$ is untrustworthy and it is possible that reflections from the boundaries could cause ejection of the molecule at this point. Simulations on larger systems are now underway to investigate if impacts at these low energies do indeed cause ejection as well or if this is just a side effect of the boundary conditions of the simulation.

\section{CONCLUSIONS}

Although at the present time we have not been able to observe coupling of the wave with sufficient energy to a neighboring fullerene to cause desorption for low energy impacts it has been demonstrated that it does occur for larger energies and that there is some indication from the simulations that there could well be a problem for low energies at which soft-landing occurs. It is felt that this could, therefore potentially, be a real problem and will almost certainly contribute to a reduction on the experimentally observed sticking probability in this kind of system. This investigation is very much preliminary at this stage.

\section{REFERENCES}

[1] DE Harrison Jr., Crit. Rev. Solid State Mater. Sci. 14, S1, 1989

[2] R Smith, K Beardmore, Thin Solid Films, 272, p255, 1996

[3] M Kerford, RP Webb, Nucl. Instrum. Meth. B, 180, p44, 2001

[4] R Smith, DE Harrison Jr., BJ Garrison, Phys. Rev. B40, p93, 1989

[5] DW Brenner, Phys. Rev. B46, p1948, 1992

[6] DW Brenner, Phys. Rev. B42, p9458, 1990

[7] R Smith, K.Beardmore, Thin Solid Films 272, p255, 1996

[8] D Christopher, R Smith, Nucl. Instrum. Meths. 180, p117, 2001

[9] RP Webb, M Kerford, E Ali, M Dunn, L Knowles, K Lee, J Mistry, F Whitefoot, Surfacd \& Interface Analysis 31, p297, 2001

[10] R Smith, RP Webb, Proc. R. Soc. Lond. A, 441, $\mathrm{p} 495,1993$ 